\title{
LOS INICIOS DE LAS GEOCIENCIAS EN LA ARQUEOLOGÍA ESPAÑOLA
}

\section{THE BEGINNINGS OF GEOSCIENCES IN THE SPANISH ARCHAEOLOGY}

\author{
José Luis RAMÍREZ-AMADOR ${ }^{1 *}$, Salvador DOMÍNGUEZ-BELLA ${ }^{1 * *}$ y Alejandro MUÑOZ MUÑOZ ${ }^{2 * * *}$ \\ ${ }^{1}$ UGEA-PHAM, Depto. Ciencias de la Tierra, Universidad de Cádiz, Puerto Real, Cádiz. Grupo HUM-440 \\ ${ }^{2}$ Doctorando. Grupo HUM-440, Universidad de Cádiz \\ * Correo electrónico: joseluisramirezamador@gmail.com \\ ** Correo electrónico: salvador.dominguez@uca.es \\ *** Correo electrónico: alejandromuozmuoz@gmail.com
}

Resumen: En la segunda mitad del siglo XX la arqueología sufre un proceso revolucionario con la implantación de nuevas metodologías de trabajo, gracias a la aplicación de nuevas disciplinas, especialmente de carácter científico, transformando la arqueología en un trabajo inter- y multidisciplinar. Esto llega a tal punto que en la actualidad se espera que todos los trabajos "serios" en arqueología se vean respaldados por datos aportados por otras disciplinas, como pueden ser estudios arqueobiológicos, geoarqueológicos, arqueométricos o dataciones por isótopos. A pesar de entender esta interdisciplinaridad en las universidades y centros de investigación, en España, como algo actual y relativamente novedoso, podemos retroceder al siglo XIX para conocer los inicios de esta interacción. En este trabajo se presenta una breve recopilación de las figuras más representativas en el mundo de las geociencias que configuraron la arqueología, y especialmente la prehistoria, en España y como encontramos en las interacciones personales, más que en las necesidades y evolución de las disciplinas, un proceso que escindió a ambas ciencias.

Palabras Clave: Geoarqueología, geociencias, Prehistoria, interdisciplinariedad, científicos españoles, historiografía.

\begin{abstract}
During the second half of the 20th century, Archeology went into a revolutionary process. The implementation of new methodologies, involving the application of new techniques from the field of Natural Sciences, allowed an improvement which turned archeology into a both interdisciplinary and multidisciplinary field. This setting leads to a situation in which, at the present time, it is expected that all "serious" works in archeology are supported by data provided in other disciplines, such as Archaeobiology, Geoarchaeology, Archaeological Studies or isotope dating. Despite the fact that this interdisciplinarity is currently and widely accepted in Universities and research centers all across Spain, this account of Archeology is still considered as quite modern-we can go back to the 19th century to know the beginnings of this interaction. This work presents a brief compilation of the most representative researchers in the world of the Geosciences that shaped Archeology. It is focused in the study of Prehistory in Spain, and it shows that the personal divergences among the main researchers on the field lead to a split between the studies on Archeology and Prehistory, which fortunately was latterly solved.
\end{abstract}

Keywords: Geoarchaeology, geosciences, Prehistory, interdisciplinarity, spanish scientists, historiography.

Sumario: 1. Los inicios de las geociencias en arqueología. 2. Las geociencias y la Prehistoria en España. 3. Conclusión. 4. Bibliografía. 


\section{Los inicios de las geociencias en arqueología}

De todas las ciencias utilizadas en la investigación arqueológica moderna, quizás, es la geología la ciencia que presenta una historia de asociación más larga con la arqueología. A pesar de ello, la geoarqueología como una disciplina reconocida dentro de esta, ha tardado en establecerse más que otras, como puede ser la bioarqueología (Little, 1995). Esta disciplina como tal se inicia a partir de 1975 con estudios sobre sedimentos arqueológicos (Shackley, 1975) y los trabajos de Davidson y Shackley (1976). Aunque la definición de geoarqueología y las competencias que abarca varían en función del país o continente (especialmente entre Europa y América del Norte), podemos entenderla como la aplicación de las geociencias para resolver problemas de investigación en arqueología (Pollard, 1999). Convencionalmente, es habitual considerar un subconjunto de geociencias incluidas dentro del término 'geoarqueología', como son la geomorfología, la estratigrafía y la sedimentología (Benito-Calvo et al., 2014). Esta interacción entre las ciencias geológicas y la arqueología puede remontarse a principios del siglo XIX, cuando la geología y la arqueología prehistórica se desarrollaron sustancialmente en paralelo. Renfrew realiza una observación que refleja la íntima relación entre ambas disciplinas, donde observa que, desde la arqueología, o al menos en la arqueología prehistórica, se recuperan casi todos los datos básicos por excavación, cada problema arqueológico comienza como un problema en geoarqueología (Renfrew, 1975). Además, la cronología, aunque básica para la arqueología y la geología, generalmente se considera una subdisciplina separada (Pollard, 1999).

Este enfoque, de estrecho paralelismo entre geología y arqueología, se ve personificado en la figura de Charles Lyell (1797-1875), considerado uno de los padres de la geología moderna, quien no solo escribió Principios de geología (1830), sino también Las evidencias geológicas de la antigüedad del hombre (1863) (Porter, 1976). Las hipótesis surgidas durante el siglo XIX con respecto a la edad de la Tierra, el mecanismo de evolución y la antigüedad de la humanidad están bien documentadas (Trigger, 1989). En la primera mitad del siglo XIX se empiezan a hallar huesos de animales extintos con hachas de mano, en un mismo contexto de acumulación, como evidencias de actividad humana en depósitos de cuevas o en las terrazas de grava de los principales ríos europeos (Lyell, 1863) (Figura 1). Como resultado de una cuidadosa observación estratigráfica de tales depósitos, la interpretación bíblica ampliamente aceptada era que estos depósitos reflejaban los restos del diluvio universal, citado en el relato bíblico de Noé, y que la Tierra había sido creada en 4004 a.C. (cita del obispo inglés J. Ussher, 1650 ), gradualmente dio paso a un modelo evolutivo, que requería períodos de tiempo considerablemente más largos. En la misma línea, los trabajos de Lyell también establecieron el principio del uniformismo en geología (Lyell, 1830), estableciendo así un largo marco cronológico para la evolución de la Tierra (Porter, 1976). En 1859, atendiendo a estas ideas, Charles Darwin plantea su modelo evolutivo para el origen de la especie humana (Puelles y Hernández, 2009), con la publicación de El origen de las especies (1859). Además, la necesidad de proporcionar un marco cronológico para tal desarrollo

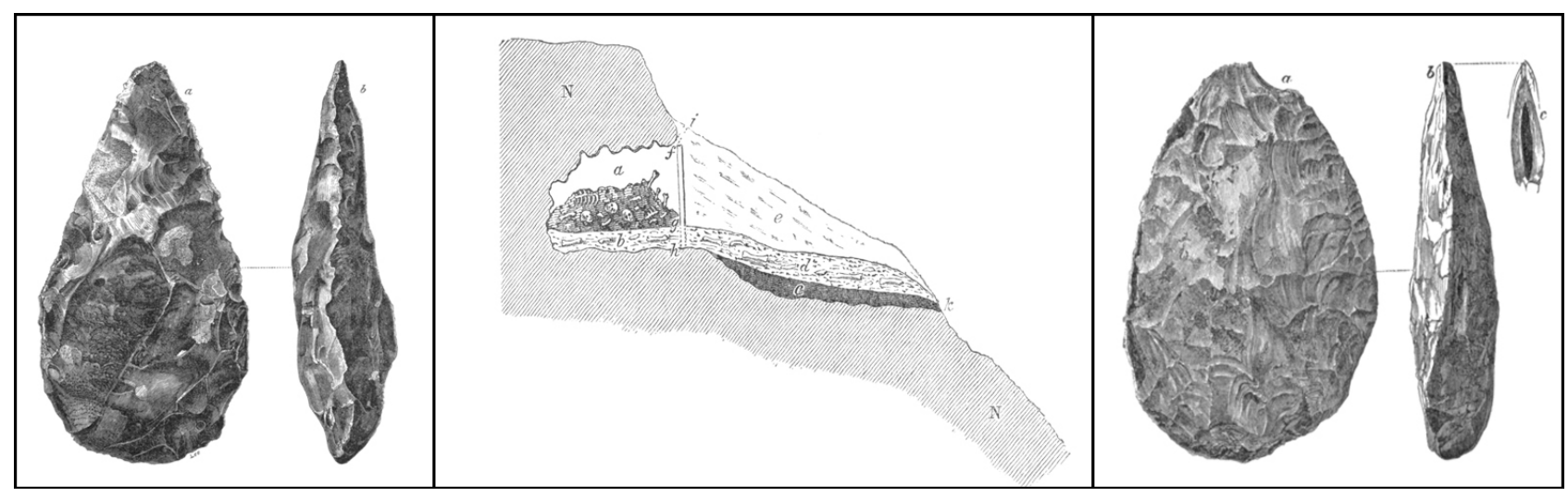

Figura 1. Algunas de las ilustraciones incluidas en el libro de Lyell Evidencias geológicas de la antigüedad del hombre, Tomo I. A la izquierda: Instrumento de sílex de St. Acheul, cerca de Amiens. En la parte central: Lugar de enterramiento en Aurignac, en el sur de Francia, que el propio autor toma de

M. Lartet. A la derecha: Implementos de sílex del Drift Post-pliocénico de Abbeville y Amiens. 
evolutivo en el contexto del avance y la retirada de la gran Edad de Hielo conduce directamente al desarrollo de la arqueología paleolítica como disciplina científica, íntimamente asociada con la geología y la paleontología (Pollard, 1999).

Desde estos primeros estudios, las geociencias y la arqueología se han apoyado mutuamente cuando el tema de estudio ha sido uno de los períodos más interesantes de la historia geológica: aquel en el que la humanidad ha desempeñado un papel cada vez mayor en la configuración del medio ambiente de la Tierra.

En la actualidad, podemos esperar una interacción cada vez mayor entre la arqueología y las geociencias, en estas y otras áreas. Los beneficios para la arqueología están, entre otros, en la vasta experiencia técnica de las geociencias. Para los geocientíficos, el beneficio radica en el aporte de un conocimiento del sistema de comportamiento humano del pasado, que será cada vez más importante a medida que avanzamos cronológicamente a través del Cuaternario. La agenda futura de las geociencias, sin duda, requiere una mejor comprensión de la relación bidireccional entre los humanos y el medio ambiente (Pollard, 1999).

En España podemos remontar estas primeras interacciones entre geocientíficos y arqueología a la primera mitad del siglo XIX.

\section{Las geociencias y la Prehistoria en España}

En España tenemos muy buenos ejemplos de esta estrecha colaboración entre geólogos y arqueólogos desde el siglo XIX, especialmente en el momento en que nace la Prehistoria española. Esta se concentra, principalmente, en la segunda mitad del siglo XIX. En un primer momento es llevada a cabo por profesores universitarios y otros profesionales, entre los que destacan los ingenieros de minas y geólogos, seguramente debido a la vinculación que la nueva disciplina arqueológica tenía con la geología y, más concretamente, con la estratigrafía (Ayarzagüena, 1992, 2018). Durante este siglo encontraremos algunos ingenieros de minas, como podemos ver en las figuras de Luis Siret (1860-1934) (Puche, 2002) o Guillermo Schulz (1800-1877) interesado por la Prehistoria en las minas asturianas, y vinculado al también ingeniero de minas Casiano de Prado (1797-1866) (Puche, 2004).

Ya desde la primera mitad del siglo XIX, cuando se inicia un especial interés por la arqueología española, empiezan a sobresalir las primeras figuras relacionadas con estas disciplinas, un claro ejemplo lo encontramos en el citado Casiano de Prado, jefe de la Comisión Permanente de Geología Industrial. Este deja patente su interés y preocupación por la preservación del patrimonio arqueológico español al remitir una circular a los jefes de distritos mineros, sobre los que tenía circunscripción, para que realizasen una búsqueda y estudio de los elementos que pudieran presentar relación con la prehistoria en las prospecciones que se realizaban sobre el terreno (De Prado, 1865). Presenta tal conocimiento en la materia, que llega a proponer la existencia de una segunda Edad de Piedra para referirse al Neolítico, periodo que había sido propuesto ese mismo año por primera vez por John Lubbock (Puche y Ayarzagüena, 1997). Destacará por ser el gran descubridor y excavador del yacimiento paleolítico de San Isidro de Madrid en 1862 (Beltrán, 2011). Otras dos figuras representativas de esta primera mitad de siglo serán Rogelio de Inchaurrandieta (1836-1915), ingeniero de caminos, que será conocido por excavar La Bastida de Totana, yacimiento arqueológico perteneciente a la cultura argárica situado en el municipio español de Totana (Ayala y Jiménez, 2002), o el de Santiago Moreno (1832-1889) (Goberna, 1986).

En mitad del siglo XIX destaca el ingeniero de caminos Eduardo Saavedra y Moragas (18291912), a quien ya le interesan épocas más recientes (Díaz-Andreu y Coltofean, 2020), será reconocido su hallazgo de los restos de la calzada romana que de Burgos se dirigía en dirección Este hacia Zaragoza. El estudio de esta vía romana le llevaría al descubrimiento de la situación de la ciudad de Numancia y que, posteriormente, le hará ser elegido académico de la Historia a los treinta y dos años (Mañas-Martínez, 2021).

A partir de los años 60 del siglo XIX sobresale Juan Vilanova y Piera (1821-1893) (Figura 2), catedrático de Geología y Paleontología de la Universidad Central desde 1853, quién adquirió una formación científica en París, en la Escuela de Minas. Destacó por ocupar en 1866 una cátedra de Prehistoria en el Ateneo de Madrid, donde impartirá docencia hasta 1881 y donde defenderá, por ejemplo, la existencia de períodos como el Mesolítico o el Calcolítico, así como su vinculación a la arqueología andaluza, especialmente en la figura de José María Tubino (1834-1888), con quien asistió a los congresos internacionales de Prehistoria de Londres y Norwich, en 1868, y de Copenhague, en 1869 (Vilanova y Tubino, 1871 en Beltrán, 2011). 

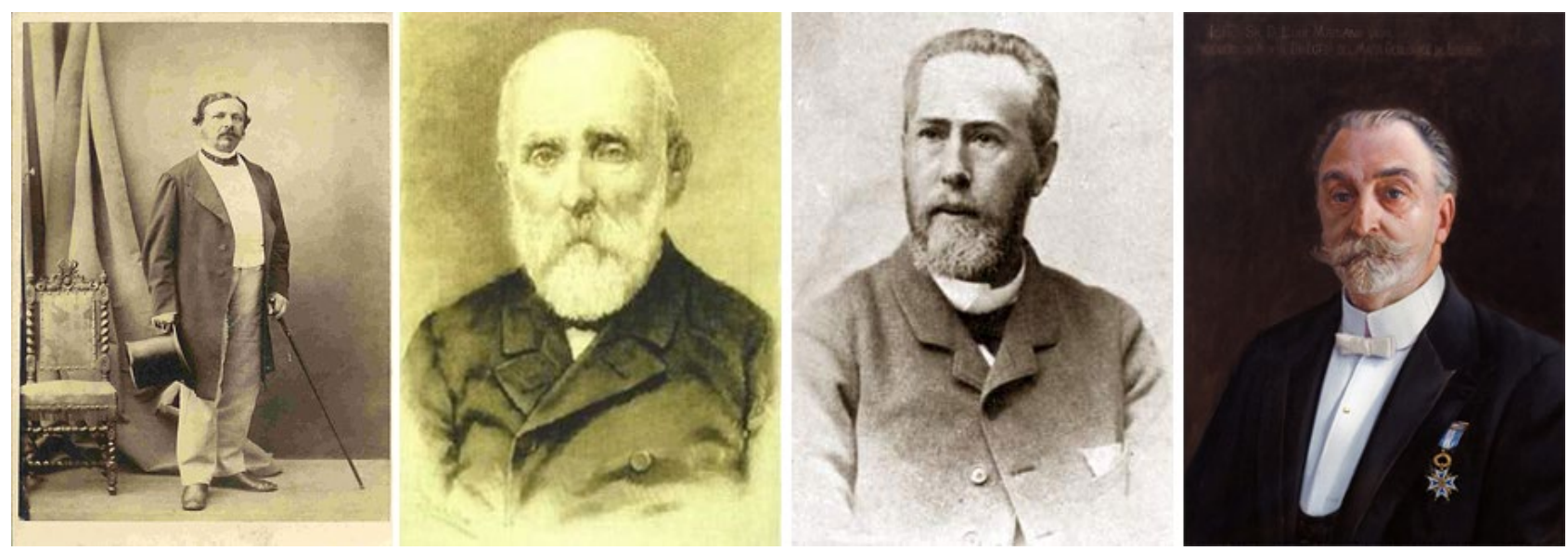

Figura 2. Algunos de los retratos más comunes de los autores. De izquierda a derecha: Antonio Machado y Núñez (1812-1896; Juan Villanova (1821-1893); José Macpherson y Hemas (1839 -1902) y por último, Luís María Vidal i Carreras (1842-1922).

En Andalucía esta relación queda especialmente reflejada en la figura del gaditano Antonio Machado y Núñez (1815-1896) (Figura 2) antropólogo, zoólogo y geólogo, catedrático en la Universidad de Sevilla, donde impartió el primer curso de Geología de España (Beltrán, 2011). Su relación complementaria con la prehistoria andaluza destacó en dos facetas; por un lado, es el creador en 1850, del Gabinete de Historia Natural en la Universidad de Sevilla, donde incluyó materiales de época prehistórica procedentes de: Alanís (Llano Moreno), El Pedroso (Sevilla); de los términos de Usagre, Don Benito y Zafra (Badajoz); mazos de minero de las antiguas explotaciones mineras de Los Silos, en Calañas (Huelva), algunos enviados por el ingeniero Recaredo de Garay, pionero de la prehistoria onubense (Orihuela, 1999), y restos antropológicos de las excavaciones de los hermanos Macpherson (Beltrán y Belén, 2007; Beltrán, 2011), que el propio Machado estudia. Por otro lado, destacó su amplia defensa del evolucionismo darwinista y su aportación a los estudios prehistóricos a partir de teorías antropológicas (Aguilar, 1990, 2002; Aguilar et al., 2010; Machado, 1869).

Dicha actividad se paraliza con el ascenso de Alfonso XII al trono y la supresión de libertad de cátedra impuesta durante su reinado (Beltrán, 2011). En Andalucía Oriental, estas nuevas teorías darwinistas recaerán en la figura de Rafael García Álvarez (1828-1894) (Puelles y Hernández, 2009). Las aportaciones de los gaditanos, ya citados, José Macpherson (1839-1902) y Guillermo Macpherson (1824-1898), consistieron en excursiones conjuntas de carácter geológico y antropológico, en las que intervendría también, el ingeniero de minas Domin- go de Orueta y Aguirre (1833-1895). De entre ellos, sobresale especialmente José (Figura 2), quien formó parte de la Sociedad Española de Historia Natural, siendo una de las figuras más destacadas de la geología española (Barrois, 1902; Martín-Escorza, 2001). En los círculos académicos es reseñable su estrecha relación con Antonio Machado y Núñez. Machado sería guía de las aficiones naturalistas de los hermanos Macpherson. Esta relación motivaría a los hermanos a practicar excavaciones en la Cueva de la Mujer, en Alhama (Alastrue, 1968). De gran importancia serán los trabajos en la realización de dos memorias de los resultados de sus excavaciones (Macpherson, 1870), una por cada campaña, y que tuvieron gran repercusión en todos los trabajos que sobre prehistoria española se realizaron en el siglo XIX. Encontró Macpherson un buen número de restos, tanto humanos como de fauna y de industria, los cuales regaló muchos de ellos al Gabinete de Historia Natural de Madrid (Beltrán, 2004).

La llegada de la llamada Revolución de 1868 trajo consigo un periodo de libertades, favoreciendo el auge de corrientes krausistas y evolucionistas darwinistas que se estaban expandiendo por Europa. Todo lo anterior provocó un aumento en el interés por las antigüedades prehistóricas, con el origen del hombre como tema de mayor controversia en la época. La prehistoria tuvo originalmente su principal acogida entre sectores liberales y progresistas de la sociedad española, asimilando la nueva teoría transformista que tanto éxito tuvo tras la publicación de El Origen de las Especies de Charles Darwin en 1859 (Beltrán, 2011).

Fuera de la universidad encontramos a Francisco María Tubino (1833-1888), gran divulgador y 
entusiasta de la prehistoria, director de la Revista de Bellas Artes, que durante 1872 impartió junto al catedrático de Geología Juan Vilanova un curso en la Universidad de Sevilla sobre "Ciencia Prehistórica" (Ayarzagüena, 1992).

Nuevas figuras, sobre todo representadas por ingenieros de minas y caminos, destacarán a final del siglo XIX. Entre ellas se encuentran Luís María Vidal i Carreras (1842-1922) (Figura 2), ingeniero de minas del Estado, que sería considerado por Manuel Cazurro como el padre de la arqueología catalana (Cazurro, 1908). Sus intervenciones estuvieron enfocadas en el hallazgo de diversas cuevas neolíticas y calcolíticas de la provincia de Lérida, tal es el caso de las cuevas del Tabaco, cerca de Camarasa; del Aigua, en la cuenca del Ribagorza; y Negra del Tragó de Noguera, en Os de Balaguer (Vidal, 1894). Además, realizó estudios sobre megalitismo (Cazurro, 1912), con resultados que han perdurado hoy día y que nos han permitido conocer muchos megalitos ya desaparecidos (Vidal, 1894). Otro importante ingeniero de minas fue Gabriel Puig y Larraz (1851-1917) quien realiza y publica un importante inventario sobre simas y cuevas en España, que es publicado en los Anales de la Sociedad Española de Historia Natural y en el Boletín Geológico y Minero, especificando aquellas que poseían yacimientos prehistóricos. Su transcendencia es tal que arqueólogos como Chaves lo utilizarían para el estudio en cuevas andaluzas o el abate Breuil, quien las utilizó de guía (Puche y Ayarzagüena, 1997). Por último, y perteneciente al mismo gremio, cabe mencionar a Augusto de Gálvez-Cañero, quien emprende en 1912 el estudio de la espeleología de la provincia de Bizkaia (Regalado y San Pedro, 2009). Es en la cueva de Balztola (Dima, Bizkaia) donde adquiere una estrecha relación con la arqueología, recogiendo fragmentos de piedras pulimentadas y cerámicas, y más de un centenar de sílex tallados que caben en el grupo citado de la clasificación de Mortillet y que serán clasificados por el abate Breuil; además de restos arqueozoológicos que este autor lleva al osteólogo francés Edouard Harlé para su estudio (Gálvez, 1913).

No sólo serán geólogos e ingenieros los encargados de realizar estudios en esta relación de geociencias y arqueología. Se puede citar, por ejemplo, a Manuel Cazurro Ruiz, que fue profesor de instituto, naturalista, coleccionista y doctorado en Ciencias Naturales (Pascual, 1976). Dentro de su faceta prehistoriadora y arqueológica destacó por aplicar sus conocimientos de estratigrafía en los trabajos realizados en Ampurias (Vallvé, 2002), en colaboración con Koenen y Schulten, además de diversas obras sobre yacimientos prehistóricos en la provincia de Gerona y sur de Francia. Entre sus publicaciones tiene gran repercusión la obra Monumentos megalíticos de la provincia de Gerona (Remesal et al., 2000).

En esta relación entre geología y arqueología, cabe resaltar a finales del siglo XIX, la figura de Luis Siret, ingeniero civil y de minas, y su hermano Enrique Siret, ingeniero de minas (Herguido, 1994). A partir de 1880 los hermanos, asentados en Almería, inician trabajos de exploración arqueológica en diferentes yacimientos (Beltrán, 2011), llegando a publicar en 1887, de forma conjunta, Les Premiers Ages du Métal dans le Sud-Est de l'Espagne (Figura 3). Convirtiéndose en una obra de primer grado en el ámbito científico (Schubart, 1979; Schubart y Arteaga, 1986), tras ello, Luis continuó siempre relacionado con el mundo de la arqueología, destacando sus aportaciones al estudio del Calcolítico almeriense.

Entre el final del XIX y principios del XX esta sinergia se prolonga. En 1907 se crea en España la Junta de Ampliación de Estudios e Investigaciones Científicas (Sánchez-Ron, 1988), mediante la formación de una élite de investigadores españoles formados en instituciones científicas extranjeras y en las escuelas y teorías predominantes, principalmente en Alemania y Francia. A partir de 1910 la Junta tendrá competencias en materia arqueológica, mediante la sección de Arqueología del Instituto de Estudios Históricos, de la que surgirá una segunda con competencias en la universidad (Beltrán, 2011; Díaz-Andreu y Coltofean, 2020). Dicho instituto no tendrá como campo de estudio la Arqueología prehistórica, dominado hasta el momento por la escuela francesa en España; para contrarrestarlo, se funda en 1912 la Comisión de Investigaciones Paleontológicas y Prehistóricas.

El desarrollo de la prehistoria en España, previo a la guerra civil, estará marcado por los constantes enfrentamientos en el campo intelectual. Los enfrentamientos fueron continuos entre Henri Breuil (1877-1961), máximo representante de la Prehistoria francesa en España, y el arqueólogo Juan Cabré (1882-1947), antiguo colaborador del abate francés y enfrentado ahora a él en el campo del estudio del arte rupestre (Beltrán y Belén, 2007). Pero es el ascenso como director de HernándezPacheco (1872-1965), catedrático de la Facultad de Ciencias (Ayarzagüena, 2018), lo que supuso el 

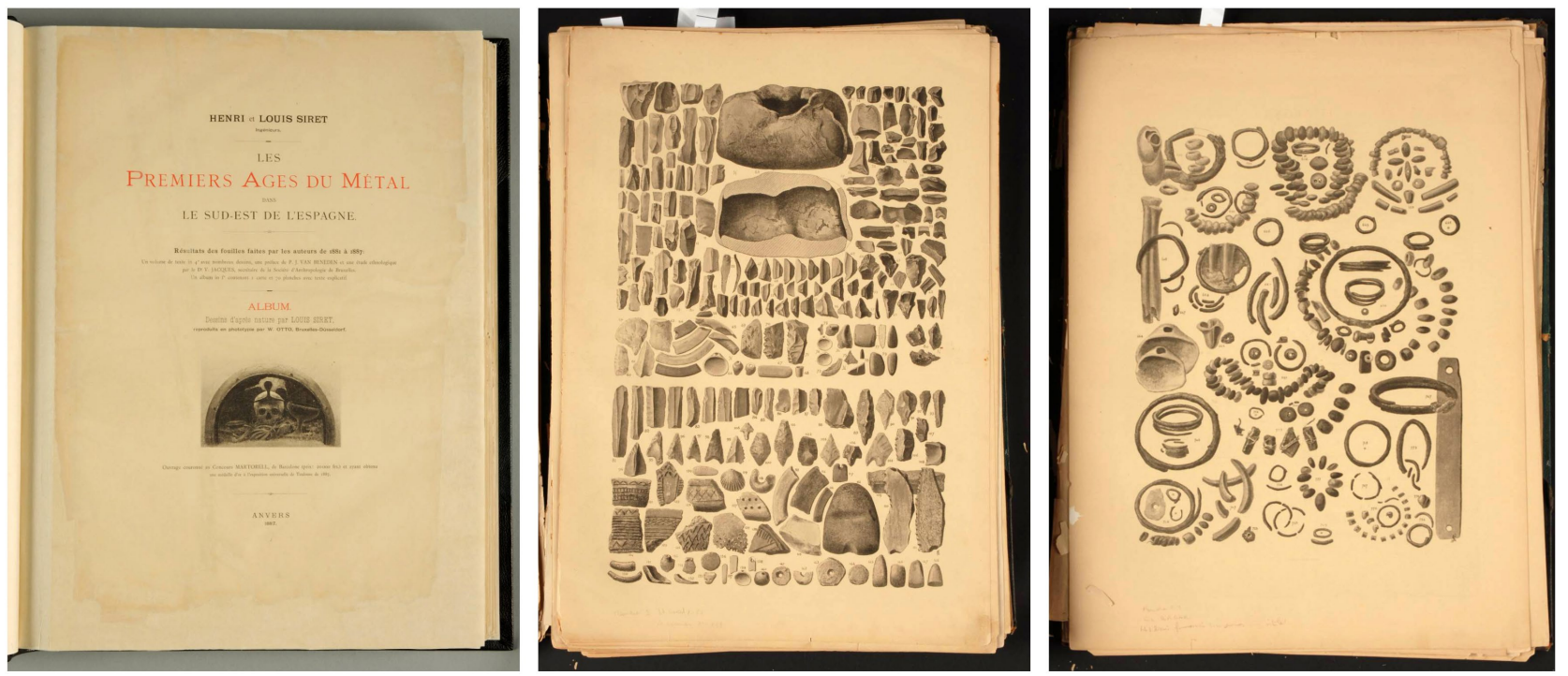

Figura 3. Algunas de las ilustraciones del libro Les Premiers Ages du Métal dans le Sud-est de L'Espagne (1887) de los hermanos Siret. Recuperado de los trabajos de restauración llevados a cabo por el centro de Intervención del Patrimonio Histórico de la Junta de Andalucía.

detonante a esta sinergia que se había presentado a lo largo de todo el siglo XIX, que se materializará en un largo enfrentamiento con Obermaier (18771946). En 1922, este pasa a ser en la Universidad Central el primer catedrático de Prehistoria en España, aunque su cátedra, titulada de Historia Primitiva del Hombre, se adscribió a la Facultad de Filosofía y Letras y no a la de Ciencias, y a pesar de que la nueva disciplina había tenido tradicionalmente una orientación naturalística más que humanística (Beltrán, 2011). Ello se debió en el fondo a intereses personales; esas rencillas determinaron en este caso el futuro de los estudios prehistóricos en la España anterior a la Guerra Civil, vinculados ya a la Facultad de Filosofía y Letras (Beltrán y Belén, 2007). Este enfrentamiento en el campo intelectual produce la escisión entre las geociencias y la prehistoria, que pasará a ser impartida durante todo el siglo XX en las facultades de humanidades y no en las de geociencias. A pesar de ello, este vínculo entre ambas disciplinas sigue vigente, manifestándose en algunas importantes figuras de la época, como ocurre en el caso del arqueólogo José Pérez de Barradas, gaditano afincado en Madrid, que realiza entre otras muchas actividades, amplios estudios sobre las terrazas cuaternarias del Río Manzanares y la prehistoria de Madrid (Figura 4)(Pérez, 1926, 1929), así como una intensa labor museística, organizando uno de los primeros museos de prehistoria en la capital (Martín, 2008) y estudios sobre América, especialmente Colombia.
Después de la Segunda Guerra Mundial, esta sinergia vuelve a producirse con la llegada de las nuevas técnicas, especialmente la radiométrica del carbono 14 (Bowman, 1990), la cual permitió comprobar que muchas de las dataciones realizadas hasta el momento eran erróneas. Estos resultados produjeron la rotura de muchos paradigmas. Además, se va a producir un cambio en la mentalidad de los arqueólogos, pues hasta el momento la mayor parte de la arqueología que se había realizado era una arqueología de tipo material, que dejaba de lado los aspectos sociales (Vargas, 1990). Los arqueólogos partían de una forma de hacer arqueología de la mano del historicismo cultural francés y alemán -difusionistas- de principios del siglo XX, momento en el que se formuló la secuencia cultural prehistórica de Mortillet, y ampliada por Henry Breuil y Denis Peyrony, a partir de una metodología estratigráfica en la que se perdió una gran cantidad de registro arqueológico. La arqueología de las escuelas francesas y alemanas imperantes en Europa era una mezcla entre la paleontología y la historia de las culturas. Childe será quien establezca, a partir de estos estudios, una sucesión estructural como las ramas de un árbol donde las culturas se influyen mutuamente.

Junto a las nuevas técnicas surge una nueva visión, así como nuevas preguntas que permiten conocer y comprender mejor las épocas pasadas. Nace así una arqueología social de la mano de un grupo de jóvenes arqueólogos unidos con el des- 


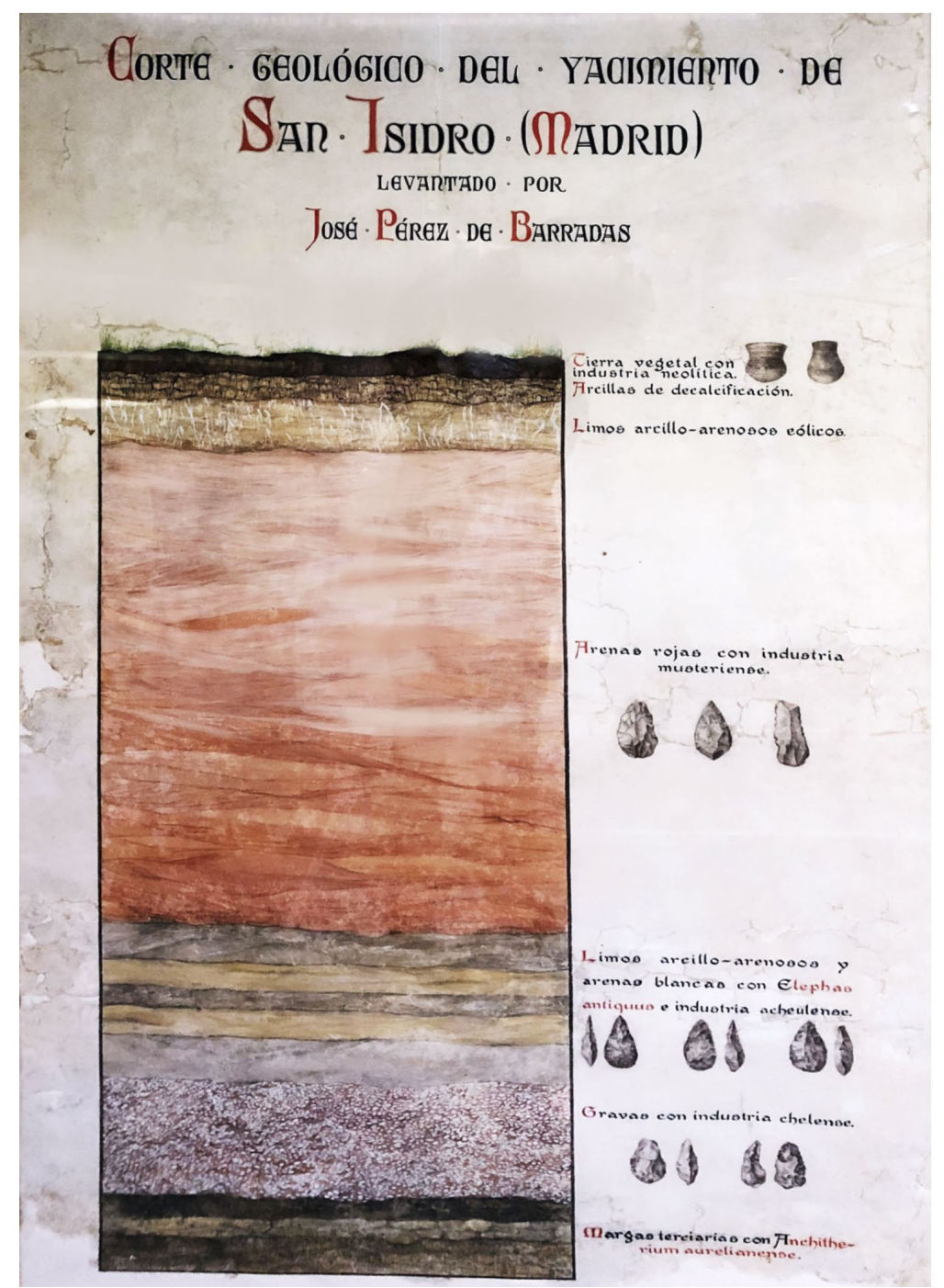

Figura 4. Corte estratigráfico del yacimiento de San Isidro, del arqueólogo Pérez de Barradas, presentado por primera vez en la exposición “Geología y Prehistoria del valle del Manzanares”, 1926.

(Ayuntamiento de Madrid. Museo de los Orígenes, № catálogo: CE1974/124/2302).

(Fuente: imagen tomada por Salvador Domínguez-Bella).

contento de la arqueología histórico-cultural por la falta de estudios científicos y antropológicos (Johnson, 2000). Muchos de los grandes autores tradicionalistas vieron como sus teorías e hipótesis eran desmontadas, suscitando grandes debates en su época bajo la mano de la nueva generación que llegaba con muchos cambios. Esa crítica se basaba en que la arqueología tradicional acumulaba materiales arqueológicos ordenándolos en una misma secuencia cultural sin crear una relación entre el objeto y el individuo que la creó (Johnson, 2000).

No es de extrañar que esa nueva visión fuese impulsada por los arqueólogos más jóvenes, entre los que cabe destacar la figura de Lewis Binford, arqueólogo estadounidense máximo representante de la tendencia arqueológica que se manifiesta en contra del Historicismo Cultural: Arqueología procesual o analítica, la New Archaeology anglosajona nacida en 1960. Esta arqueología, por definición, se considera prácticamente funcionalista (Hodder, 1982). La nueva arqueología defiende el método científico, y considera a los grupos humanos como sistemas culturales con comportamientos sujetos a la interacción de una cultura con el medio ambiente (Hodder, 1982, 1998). Además, poseían objetos materiales e inmateriales, por lo que no es de extrañar la publicación de trabajos sobre una base de antropología social de las culturas, donde tendrá un papel importante la etnología y la arqueología experimental, con metodologías científicas. Binford, en 1965, y Clarke en 1968, aplicaron la Teoría General de Sistemas, planteada 
ya antes por Lelie A. White, que defiende que la cultura es un sistema integrado por subsistemas relacionados (Hernando, 1992; Hodder, 1998). La obra que mejor representa la aplicación de este sistema es la Tesis Doctoral de Renfrew, en 1972, La emergencia de la Civilización del Egeo.

Renfrew es también quién modificará y modernizará el sistema de estudio arqueológico dirigiéndolo hacia una nueva versión procesual-cognitiva donde se sigue manteniendo la necesidad del método científico para conocer el pasado, métodos hipotéticos-deductivos, estudios objetivos, rechazo de convicciones políticas, etc. (Renfrew, 1989). La rigurosidad y la mayor cantidad de datos son arrojados por la nueva tendencia arqueológica, con un fuerte carácter interdisciplinar y multidisciplinar que les permitió imponerse frente a la arqueología tradicional, de la cual criticaban su carencia científica a la hora de trabajar, transformándose así en una actividad de carácter científico. De esta forma, nace la arqueología moderna, donde aparecen, entre otras, la geoarqueología y la arqueometría como disciplinas fundamentales en el estudio de materiales del pasado. Aunque, a pesar de todos estos cambios, el historicismo cultural aún se mantuvo con fuerza en Europa (Hernando, 1992).

\section{Conclusión}

Como se ha podido ver la arqueología española y las geociencias presentan una fuerte vinculación ya desde inicios del siglo XIX. A lo largo de este periodo destaca la vinculación de importantes figuras españolas del mundo de las geociencias como Antonio Machado y Núñez, Guillermo Mcpherson o Luis Siret, entre muchos otros. Es especialmente la prehistoria la disciplina que más se vincula a estos profesionales. El desarrollo de los acontecimientos permitía prever el desarrollo de la prehistoria como una rama que evolucionara dentro de las facultades de geociencias, al menos en el caso español. Todo ello entendido gracias al impulso del nacimiento de las teorías evolucionistas que van apareciendo a lo largo de este mismo siglo, que a su vez se nutren de la aparición un siglo antes de las ideas geológicas de una Tierra cambiante.

La ruptura de esta sinergia, producida a finales del siglo XIX y principios del XX, está más relacionada con rencillas personales que con la propia evolución de la disciplina, punto de inflexión que sitúa a la prehistoria, a partir de ese momento, en las facultades de humanidades y letras.
A pesar de ello, los avances en los últimos años de la arqueología, y las técnicas interdisciplinares vinculadas a ella, están arrojando una gran cantidad de datos. Estos, que día a día son más fiables y precisos, están produciendo fuertes sacudidas en los paradigmas planteados desde hace décadas. Dichos cambios están transformando la arqueología actual, la cual ya no puede entenderse sin estas ciencias multidisciplinares, entre las que podemos situar la geoarqueología.

\section{Bibliografía}

AGUILAR CRIADO, Encarnación; ARROYO, Juan, FIERRO, Elena; JORDANO, Pedro (coords.). 2010: Darwin en Sevilla. Antonio Machado y Núñez y los darwinistas sevillanos, Universidad de Sevilla. Sevilla.

AGUILAR CRIADO, Encarnación. 1990: Cultura Popular y Folklore en Andalucía (Los orígenes de la Antropología). Diputación de Sevilla. Sevilla. AGUILAR CRIADO, Encarnación. 2002: “Arqueólogos y antropólogos sevillanos a finales del siglo XIX". En B. DEAMOS y B. FORTES (eds.): $A r-$ queología fin de siglo. La Arqueología española de la segunda mitad del siglo XIX (I Reunión Andaluza de Historiografía Arqueológica), pp. 89-102. Universidad de Sevilla. Sevilla.

ALASTRUE, Eduardo. 1968: La personalidad y la obra de Macpherson (1839-1902). Publicaciones de la Universidad de Sevilla. Sevilla.

AYALA JUAN, María Manuela; JIMÉNEZ LORENTE, Sacramento. 2002: "Emeterio Cuadrado Díaz y el sudeste peninsular hace cuatro mil años. La cultura del Argar". AnMurcia, 16-17, pp. 67-72. AYARZAGÜENA SANZ, Mariano. 1992: La Arqueología Prehistórica y Protohistórica Española en el siglo XIX. UNED. Madrid

AYARZAGÜENA SANZ, Mariano. 2018: "El papel desempeñado por geólogos e ingenieros de minas en la consolidación de la Arqueología prehistórica en España (1860-1970)". Boletín Geológico y Minero, 129 (1/2), pp. 3-33.

BARROIS, Charles. 1902: "Notice necrológique sur José Mac-Pherson". Ann. Soc. Géol. du Nord, XXXI, pp. 312-317.

BELTRÁN FORTES, José. 2011: "Historia de la arqueología andaluza de 1860 a 1939: en el marco vital de Luis Siret (1860-1934)". En: Memorial Luis Siret: la tutela del patrimonio prehistórico, pp. 25-38. Junta de Andalucía, Consejería de Cultura. Antequera. 
BELTRÁN FORTES, José; BELÉN DEAMOS, María. 2007: "La Arqueología en la Universidad de Sevilla. 1. El siglo XIX". En B. DEAMOS y B. FORTES (eds.): Las instituciones en el origen y desarrollo de la Arqueología en España, pp. 93-142. Universidad de Sevilla. Sevilla.

BENITO-CALVO, Alfonso; LOZANO, Isidoro Campaña; KARAMPAGLIDIS; Theodoros. 2014: “Conceptos básicos y métodos en geoarqueología: geomorfología, estratigrafía y sedimentología". Treballs d'Arqueologia, 20, pp. 41-54.

BOWMAN, Sheridan. 1990: Radiocarbon Dating. British museum Pub. Londres.

CAZURRO, Manuel. 1908: "Las cuevas de Serinyà y otras estaciones prehistóricas de NE. de Cataluya". Anuari de l'IEC, pp. 43-88.

CAZURRO, Manuel. 1909: "Fragments de vasos ibèrics d'Empúries". Anuari de l'IEC, 11, pp. 551-562.

CAZURRO, Manuel. 1912: Monumentos megalíticos de la provincià de Cerona, Junta para la ampliación de Estudiós é Investigaciones Científicas, Centro de Estudiós Históricos. Madrid.

CAZURRO, Manuel. 1913: Guía ilustrada de las ruinas de Ampurias y Costa Brava catalana (editada e ilustrada por José Esquirol). L'Escala. Barcelona.

DARWIN, Charles. 1959: The origin of species. John Murray. Londres.

DAVIDSON, Donald; SHACKLEY, Myra. 1976: Geoarchaeology: Earth Science and the Past. Duckworth. Londres.

DE PRADO, Casiano. 1865: "Circular de la Comisión Permanente de Geología Industrial, dirigida a los ingenieros de minas, Jefes de las Provincias". Revista Minera, XVI (357), pp. 25-236.

DÍAZ-ANDREU, Margarita; COLTOFEAN, Laura. 2020: "Hacia una historia de la interdisciplinariedad en la Arqueología española: introduciendo una nueva perspectiva". Veleia, 37, pp. 3-32.

GÁLVEZ CAÑERO, Augusto. 1913: "Nota acerca de las cavernas de Vizcaya". Boletín Instituto Geológico de España, XXXIII, pp. 184-198.

GOBERNA, María Victoria. 1986: "Los estudios de Prehistoria durante la segunda mitad del siglo XIX y primeros años del XX - La obra de Luis Siret". En: Homenaje a Luis Siret, pp. 28-34. Junta de Andalucía. Sevilla.

HERGUIDO, Carlos. 1994: Apuntes y documentos sobre Enrique y Luis Siret ingenieros y arqueólogos. Instituto de Estudios Almerienses.Al- mería.

HERNANDO GONZALO, Almudena. 1992: "Enfoques teóricos en Arqueología”. SPAL, 1, pp. 1135.

HODDER, Ian. 1982: Theoretical archaeology: a reactionary view. En I. HODDER (ed.): Symbolic and structural Archaeology, pp.1-16. Crambridge University Press. Cambridge .

HODDER, Ian. 1998: Interpretación en Arqueología. Corrientes actuales. Crítica. Barcelona.

JOHNSON, Matthew. 2000: Teoría arqueológica. Una introducción. Ariel Historia. Barcelona.

LITTLE, Michael. 1995: "Adaptation, Adaptability and Multidisciplinary Research". En: N.T. BOAZ y L. WOLFE (eds.): Biological Anthropology. The State of the Science, pp. 121-148. International Institute for Human Evolutionary Research. Oregon.

LYELL, Charles. 1830: Principles of Geology 1st edition, 1st vol. Jan. 1830. John Murray. Londres.

LYELL, Charles. 1863: Geological Evidences of the Antiquity of Man 3st edition, revised. John Murray. Londres.

MACHADO Y NÚÑEZ, Antonio. 1869: "Congreso Internacional de Antropología Prehistórica". Revista de Filosofía, Literatura y Ciencias de Sevilla, I, pp- 3-39 y 281-287.

MACPHERSON, Guillermo. 1870: La cueva de la Mujer. Descripción de una caverna conteniendo los restos prehistóricos, descubierta en las inmediaciones de Alhama de Granada. Imprenta de la Revista Médica de Federico Joly y Velasco. Cádiz.

MACPHERSON, Guillermo. 1876: Los habitantes primitivos de España. Impresores de Cámara. Madrid.

MAÑAS MARTÍNEZ, Julián. 2021: "Eduardo Saavedra Moragas". Real Academia de la Historia, Diccionario Biográfico electrónico. https:// dbe.rah.es/biografias/5527/eduardo-saavedra-moragas. Consultado el 25 de octubre de 2021.

MARTÍN ESCORZA, Carlos. 2001: “Vida y obra de español José Macpherson Hemas (Cádiz 1839La Granja 1902)". Bol. Com. Hist. Geol. Esp, n.음 16, pp. 3-7.

MARTÍN FLORES, Alfonso. 2008: "Los Museos Prehistóricos de Pérez de Barradas". En Arqueología, América, Antropología. José Pérez de Barradas 1897-1981. Catálogo de la Exposición. 12 junio-30 noviembre, pp. 243-285. Museo de los Orígenes. Casa de San Isidro. Madrid. 
ORIHUELA, Antonio. 1999: Historia de la Prehistoria: el suroeste de la península ibérica. Diputación de Huelva. Huelva.

PASCUAL, Pompeu. 1976: El professor Don Manuel Cazurro i Ruiz i l'Institut de Girona del seu temps. Quiaderns De Prehistoria I Arqueologia. Gerona.

PÉREZ DE BARRADAS, José. 1926: "Madrid prehistórico". En Sociedad Española de Amigos del Arte: Exposición del antiguo Madrid. Catálogo general ilustrado, pp. 266-268. Gráficas Reunidas. Madrid.

PÉREZ DE BARRADAS, José. 1929: "Yacimientos prehistóricos de los alrededores de Madrid". Boletín del Instituto Geológico y Minero de España, vol. LI, pp. 153-172.

POLLARD, Mark (ed.). 1999: Geoarchaeology: exploration, environments, resources. The Geological Society of London. Londres.

PORTER, Rodney. 1976: "Charles Lyell and the principles of the History of Geology". British Journal of History of Science, 22, pp. 91-103

PUCHE RIART, Octavio. 2002: "La contribución de los ingenieros a la Arqueología española". En S. QUERO y A. PÉREZ (eds.): Historiografía de la Arqueología Española: las Instituciones, pp. 13-45. Museo de San Isidro. Madrid.

PUCHE RIART, Octavio. 2004: "Casiano De Prado y Valle". En M. AYARZAGÜENA y G. MORA (eds): Pioneros de la Arqueología en España del siglo XVI a 1912, pp. 79-87. Museo Arqueológico Regional. Alcalá de Henares.

PUCHE RIART, Octavio; AYARZAGÜENA, Mariano. 1997: "Ingenieros de minas arqueólogos en el siglo XIX. La huella De Prado. Homenaje a Casiano de Prado (1797-1866) En el bicentenario de su nacimiento". Boletín Geológico y Minero, vol. 108-3, pp. 295-330.

PUELLES, Manuel; HERNÁNDEZ, Margarita. 2009: "El darwinismo en los manuales escolares de ciencias naturales de segunda enseñanza desde la publicación del "Origen de las especies" en España hasta finales del siglo XIX". Anuario de Historia de la Iglesia, 18, pp. 69-84.

REGALADO BUENO, Encarnación; SAN PEDRO CALLEJA, Ziortza. 2009: Informe de Excavación arqueológica: excavación arqueológica en la cueva de Balzola. 1a campaña: 2008 proyecto Balzola 2008-2012, pp. 7-8. Balzola. Dima.

REMESAL RODRÍGUEZ, José; AGUILERA MARTÍN, Antonio; PONS PUJOL, Luís. 2000: Comisión de Antigüedades de la Real Academia de la Histo- ria. Cataluña. Catálogo e Índices. Real Academia de la Historia. Madrid.

RENFREW, Colin. 1975: "Trade as action at a distance: questions of integration and communication". En J. SABLOFF y C. LAMBERG-KARLOVSKY (eds.): Ancient Civilization and Trade, pp. 3-59. University of New Mexico Press. Albuquerque.

RENFREW, Colin. 1989: "Comments to Archaeology into the 1.990s". Norwegian Archaeological Review, 22, №1, pp. 33-41

SÁNCHEZ-RON, José Manuel. 1988: La Junta para la Ampliación de Estudios e Investigaciones Científicas 80 años después (1907-1987). Consejo Superior de Investigaciones Científicas. Madrid.

SCHUBART, Hermanfrid. 1979: Nuevas fuentes para la cultura del Argar, XV Congreso Nacional de Arqueología (Lugo, 1977)". Zaragoza.

SCHUBART, Hermanfrid; ARTEAGA, Oswaldo. 1986: "El mundo de las colonias fenicias occidentales". En: Homenaje a Luis Siret, pp. 499525. Junta de Andalucía. Sevilla.

SHACKLEY, Myra. 1975: Archaeological Sediments. A survey on analytical methods. Butterworths. Londres.

TRIGGER, Bruce. 1989: A History of Archaeological Thought. Cambridge University Press. Cambridge.

USSHER, James. 1650: Annales Veteris Testamenti, a prima mundi origine deducti, una cum rerum Asiaticarum et Aegyptiacarum chronico, a temporis historici principio usque ad Maccabaicorum initia producto.

VALLVÉ, Isabel. 2002: Manuel Cazurro Ruiz: la seva collecció arqueológica i la seva vinculació amb Empúries. Tesis doctoral. Universidad de Gerona.

VARGAS, Iraida. 1990: Arqueología, ciencia y sociedad. Abre Brecha. Caracas.

VIDAL Y CARRERAS, Luis Mariano. 1894: "Coves prehistóricas de la provincia de Lleida". Conferencia impartida en el Centro Excursionista de Cataluña el dia 29 de mayo de 1894". Butlletí del Centre Excursionista de Catalunya, 13 (4), pp. 81-108.

VILANOVA Y PIERA, Juan; TUBINO Y OLIVA, Francisco María. 1871: Viaje científico a Dinamarca y Suecia con motivo del Congreso Internacional Prehistórico, celebrado en Copenhague en 1869. Ministerio de Fomento. Madrid. 